\title{
Experiments with the Double Solenoid System RIBRAS
}

R. Lichtenthäler ${ }^{a *}$, R. Pampa Condori ${ }^{a}$, A. Lépine-Szily ${ }^{a}$, M. A. G. Alvarez ${ }^{a}$, L. R. Gasques $^{a}$, J. Artech ${ }^{b}, \mathbf{R}$. Arteche ${ }^{b}$, O. B. de Morais ${ }^{a}$, V. Guimarães ${ }^{a}, \mathbf{N}$. Deshmukh $^{a}$, D. R. Mendes Junior ${ }^{c}$, K. C. C. Pires ${ }^{a d}$, P. N. de Faria ${ }^{a}$, E. Leistenschneider ${ }^{a}$, V. Scarduelli ${ }^{a}$.

${ }^{a}$ Departamento de Física Nuclear, Universidade de São Paulo, C.P. 66318, 05389-970, São Paulo, Brazil

${ }^{b}$ Centro de Aplicaciones Tecnológicas y Desarollo Nuclear (CEADEN), Cuba

${ }^{c}$ Instituto de Física, Universidade Federal Fluminense, Niterói, RJ, 24210-340, Brazil

${ }^{d}$ Universidade Tecnológica Federal do Paraná, campus Cornélio Procópio, PR, 86300-000, Brazil.

rubenseif.usp.br

A description of the Radioactive Ion Beams in Brasil (RIBRAS) facility and the result of some experiments recently performed with the double solenoid system will be presented.

X Latin American Symposium on Nuclear Physics and Applications (X LASNPA),

1-6 December 2013

Montevideo, Uruguay

${ }^{*}$ Speaker. 


\section{Introduction}

The possibility of producing secondary beams of nuclei out of the line of stability opened a huge field of research in low energy nuclear physics. In the 1960s there were about 1200 known nuclides whereas nowadays, more than 3500 were produced in laboratory, and this number is increasing quickly. The study of nuclei out of the line of stability raised new interesting questions in nuclear physics, as for instance, what are the limits of the existence of nuclei in the nature? The neutron and proton drip lines are defined as the limits, beyond which no bound nuclei is found. The exact position of the drip lines in the nuclear chart have not yet been determined. For nuclei near to the neutron drip line, interesting new phenomena have been observed. Some light neutron rich nuclei such as ${ }^{6,8} \mathrm{He},{ }^{11} \mathrm{Be},{ }^{11} \mathrm{Li}$ and others present a pronounced cluster structure, formed by a more or less stable core, surrounded by a neutron halo or skin, which ex tends over large distances from the core, and whose density is much smaller than the normal nuclear matter. Due to this halo, those nuclei present a much higher reaction cross section, compared to the normal tightly bound nuclei and even to the stable weakly bound isobar nuclei.

In addition, nuclei like ${ }^{11} \mathrm{Li}$ and ${ }^{6} \mathrm{He}$ are examples of 3-body systems [1,2], in which only the three components $(n+n+$ core) form a bound system and, any two-body subsystems ( $n n$ or $n+$ core) is unbound. These nuclei may be considered as 'laboratories' for the study of three body forces, which play an important role in the physics of many body systems.

The role of nuclear physics in astrophysics is crucial $[3,4]$ and the existence of long lived exotic nuclei may also have an important impact in astrophysics. For instance, the presence of light exotic nuclei such as ${ }^{6,8} \mathrm{He}$ in supernova explosions, could act as a trigger for the synthesis of heavier elements, helping to overcome the instability $A=5,8$ gaps [5]. Moreover, the existence of the neutron halo might have an effect on the nuclear structure models, as the quenching of the shell gaps or even the existence of new magic numbers. The possibility of modifications in the shell structure of medium masses nuclei could have consequences for the problem of the synthesis of elements heavier than Iron (r-process), which is one of the main challenges in nuclear astrophysics nowadays.

Several laboratories over the world have been dedicated to the research using radioactive ion beams (RIB). In São Paulo, Brasil, the Radioactive Ion Beams in Brasil (RIBRAS) [6,7] facility is operating since 2004, and producing secondary beams of light exotic nuclei such as ${ }^{8} \mathrm{Li},{ }^{6} \mathrm{He}$, ${ }^{7} \mathrm{Be}$ and others. An extense research program has been developed since then, mostly on elastic scattering measurements [8-18] but also on reactions $[19,20]$. In the present contribution we will present some recent results.

\section{The RIBRAS system and the production of RIB}

Most of the large laboratories over the world produce secondary beams in the intermediate energy region (30 MeV/A up to $1 \mathrm{GeV} / \mathrm{A}$ ). At these energies RIB are mainly produced via nuclear fragmentation reactions between a high power primary beam and a target. Two methods are available to produce RIB, in-flight and ISOL. In the in-flight method, the secondary particles are produced by nuclear reactions between the primary beam and a thin primary target. The reaction products are selected in-flight by electromagnetic isotope separators such as dipoles, solenoids or 


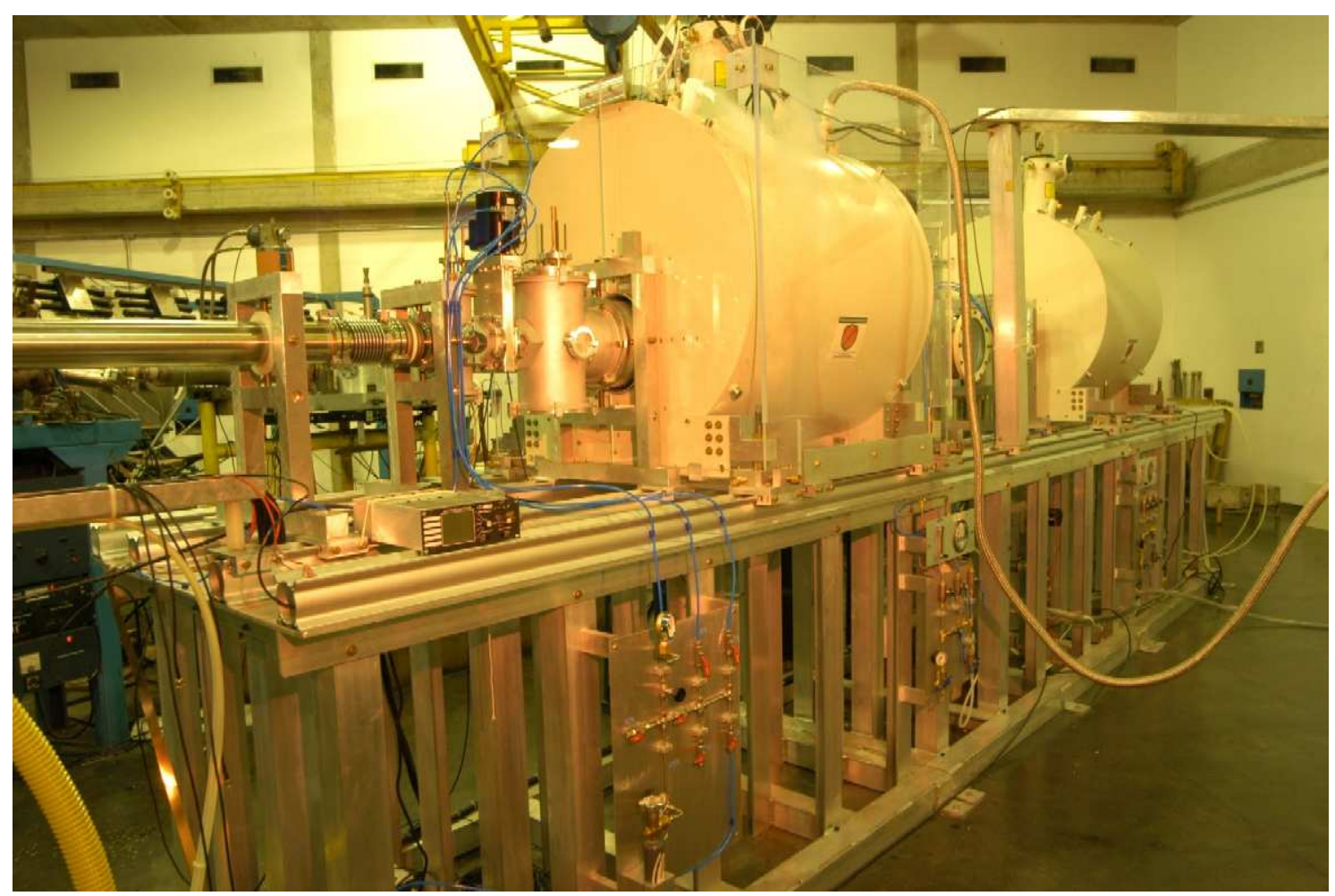

Figure 1: Photo of the RIBRAS system.

Wien filters or a combination of these elements. In this case, the secondary beam has characteristics such as emmitance and momentum distribution, which are determined by the energy loss and momentum transfer processes in the primary target. In many cases, the in-flight method provides intense secondary beams but contaminated with particles of the same magnetic rigidity.

The ISOL method, on the other side, uses a primary target sufficiently thick to completely stop the primary beam particles. The secondary particles diffuse through the hot matter of the target to an ion source where they are re-ionized and injected in a secondary accelerator. In general, the ISOL method produces secondary beams of much better quality, concerning the emmitance and energy resolution, but with lower intensity than the in-flight method.

RIBRAS uses the in-flight method and two superconducting solenoids as separators. The driver accelerator is a $8 \mathrm{MV}$ Tandem which delivers primary beams of protons, $\mathrm{Li}, \mathrm{B}, \mathrm{Be} \mathrm{C}, \mathrm{O}$, up to $\mathrm{Cu}$ with energies in the range $3-5 \mathrm{MeV} / \mathrm{A}$ and intensities of $0.3-5 \mu \mathrm{Ae}$.

In figure 1 we show a photo and in figure 2 we show a scheme of the RIBRAS system.

The primary beam comes from the left side and the secondary particles are produced in the primary target (1) by nuclear reactions. ${ }^{6,7} \mathrm{Li}$ and ${ }^{10,11} \mathrm{~B}$ are the most common primary beams used so far in RIBRAS, with intensities ranging between $0.2-0.6 \mu \mathrm{Ae}$. The standard primary target is a ${ }^{9} \mathrm{Be}$ foil of $8-12 \mu \mathrm{m}$ thickness but gas targets such as ${ }^{3} \mathrm{He}$ and others have also being used. The primary beam is suppressed by a Faraday cup (3), connected to a current integrator module which provides the total incident charge.

The first solenoid makes a $B \rho=\frac{\sqrt{2 m E}}{q}$ selection of the particles produced in the primary 


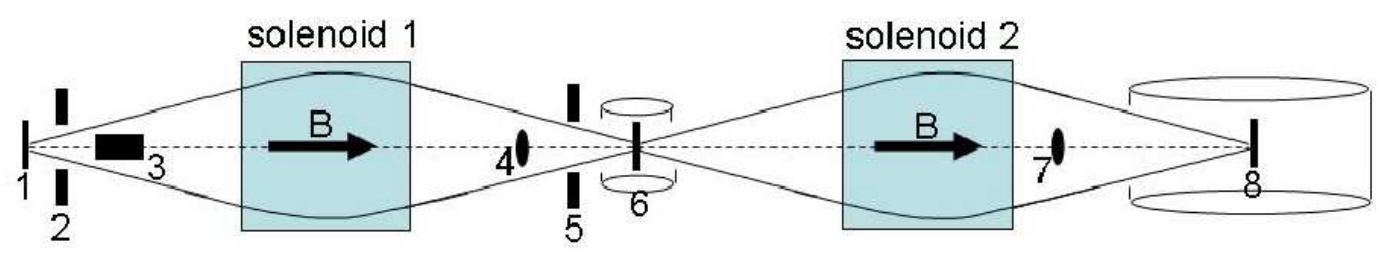

Figure 2: Scheme of the double solenoid system. 1- primary target, 2 and 5 collimators, 3-Faraday cup, 4 and 7 blockers, 6- mid-way scattering chamber, 8-secondary scattering chamber.

target that are accepted by the $30 \mathrm{msr}$ bore. Particles with the same $B \rho$ are focused in the ISO250 intermediate scattering chamber and, unwanted particles are stopped by the collimators and blockers strategically positioned over the beam line.

In Figure 3 we show a spectrum of the cocktail beam for the ${ }^{9} \mathrm{Be}\left({ }^{7} \mathrm{Li},{ }^{8} \mathrm{Li}\right){ }^{8} \mathrm{Be}$ production reaction, measured in the intermediate scattering chamber. We see the ${ }^{8} \mathrm{Li}$ leading beam peaks and the contaminants peaks ${ }^{7} \mathrm{Li}^{2+},{ }^{6} \mathrm{He},{ }^{4} \mathrm{He}$, and triton, deuteron and protons. The ${ }^{8} \mathrm{Li}$ peak is doubled due to an excited state at $0.98 \mathrm{MeV}$. The excite state peak can be completly blocked in the lollipop by a proper solenoid tunning, as shown in the insert of Figure 3.

In 2011 we installed the secondary scattering chamber $(70 \mathrm{~cm}$ diameter, $40 \mathrm{~cm}$ height $)$ after the second solenoid. The first experiment was performed using a ${ }^{6} \mathrm{He}$ beam [21], produced by the ${ }^{9} \mathrm{Be}\left({ }^{7} \mathrm{Li},{ }^{6} \mathrm{He}\right){ }^{10} \mathrm{~B}$ production reaction. In figure 4 we show spectra of the beam in the intermediate ISO-250 chamber and in the secondary scattering chamber after the second solenoid selection. An $\mathrm{CH}_{2}$ foil of $12 \mu \mathrm{m}$ was used as absorber in the intermediate scattering chamber, in order to allow an additional $B \rho$ selection by the second solenoid. The purity of the ${ }^{6} \mathrm{He}$ beam is improved from $16 \%$ in the intermediate scattering chamber to $92 \%$ in the secondary chamber after the second solenoid selection.

\section{Experiments with the double solenoid system}

Since its inauguration in 2004, several experiments have been performed in RIBRAS, mainly elastic scattering measurements of ${ }^{6} \mathrm{He},{ }^{8} \mathrm{Li}$ and ${ }^{7} \mathrm{Be}$ on different mass targets $[9,11,12,14,15,18]$. Those experiments have been carried out in the intermediate scattering chamber, using only one solenoid as selector. At this point, the secondary beam is contaminated with other particles as shown in figure 3 , but it is sufficiently clean for elastic scattering measurements.

The production of ${ }^{6} \mathrm{He}$ and ${ }^{8} \mathrm{Li}$ secondary beams, with improved purity, due to the second solenoid selection, opens the possibility to perform more sophisticated experiments. For instance, measurements of resonant elastic scattering by the thick target method [21,22]. In this method, a thick $12 \mu m \mathrm{CH}_{2}$ (polyethylene) foil is used as secondary target. The foil is choosen to be sufficient thick to completly stop the secondary beam particles, however, the recoil protons lose little energy in the foil and go through the target up to detectors placed in the forward hemisphere region. As the energy of the recoil protons can be related to the energy of the incident particle in the position where 


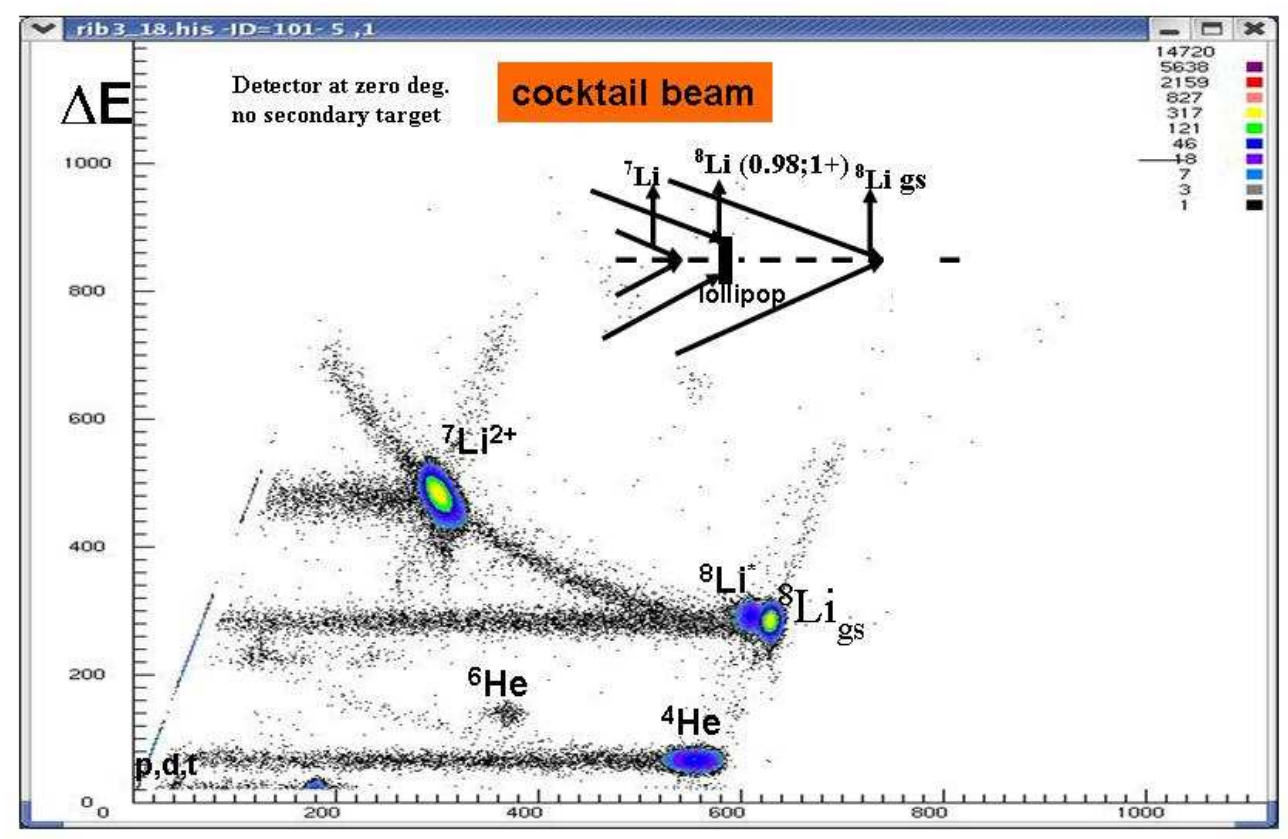

Figure 3: Spectrum of the cocktail beam in the intermediate scattering chamber.

the collision took place, the measurement of the energy spectrum of the protons provide an entire excitation function measurement. Peaks in the proton spectrum are then related to resonances in the compound system (projectile+target). Measurements with a pure carbon target can be performed for background subtraction, corresponding to reactions in the carbon of the polyethylene foil.

As the secondary beam is supressed in the target, the detectors can be placed at very forward angles, including zero degrees, which corresponds to $180 \mathrm{deg}$ in the center of mass system.

Such kind of measurements provide information of the position, and the total and partial widths of excited states in the compound nucleus, in an excitation energy region, which is not easily accessed with beams of stable nuclei. Angular distribution measurements allow to extract the angular momentum of the resonances.

In figure 5, we present spectra of the light particles emmited from the collision between a ${ }^{6} \mathrm{He}$ beam and a thick $\mathrm{CH}_{2}$ target placed in (8), see figure 2. Data have been obtained at three angles, zero, 20 and $25 \mathrm{deg}$ which correspond to $\theta_{c m}=180,140$ and 130 deg respectively for the ${ }^{6} \mathrm{He}(p, p){ }^{6} \mathrm{He}$ scattering. At zero degrees, we observe that there are still contaminant peaks from remnant secondary beams of alphas, tritons and deuterons. However, those contaminants practically disappear at larger angles and a clear proton line is observed. In the region indicated as proton line in figura 5 there is a peak corresponding to the $11.24 \mathrm{MeV}$ excited state of ${ }^{7} \mathrm{Li}$, which is the isobaric analog state of the ground state of the ${ }^{7} \mathrm{He}[25]$. 
1 solenoid

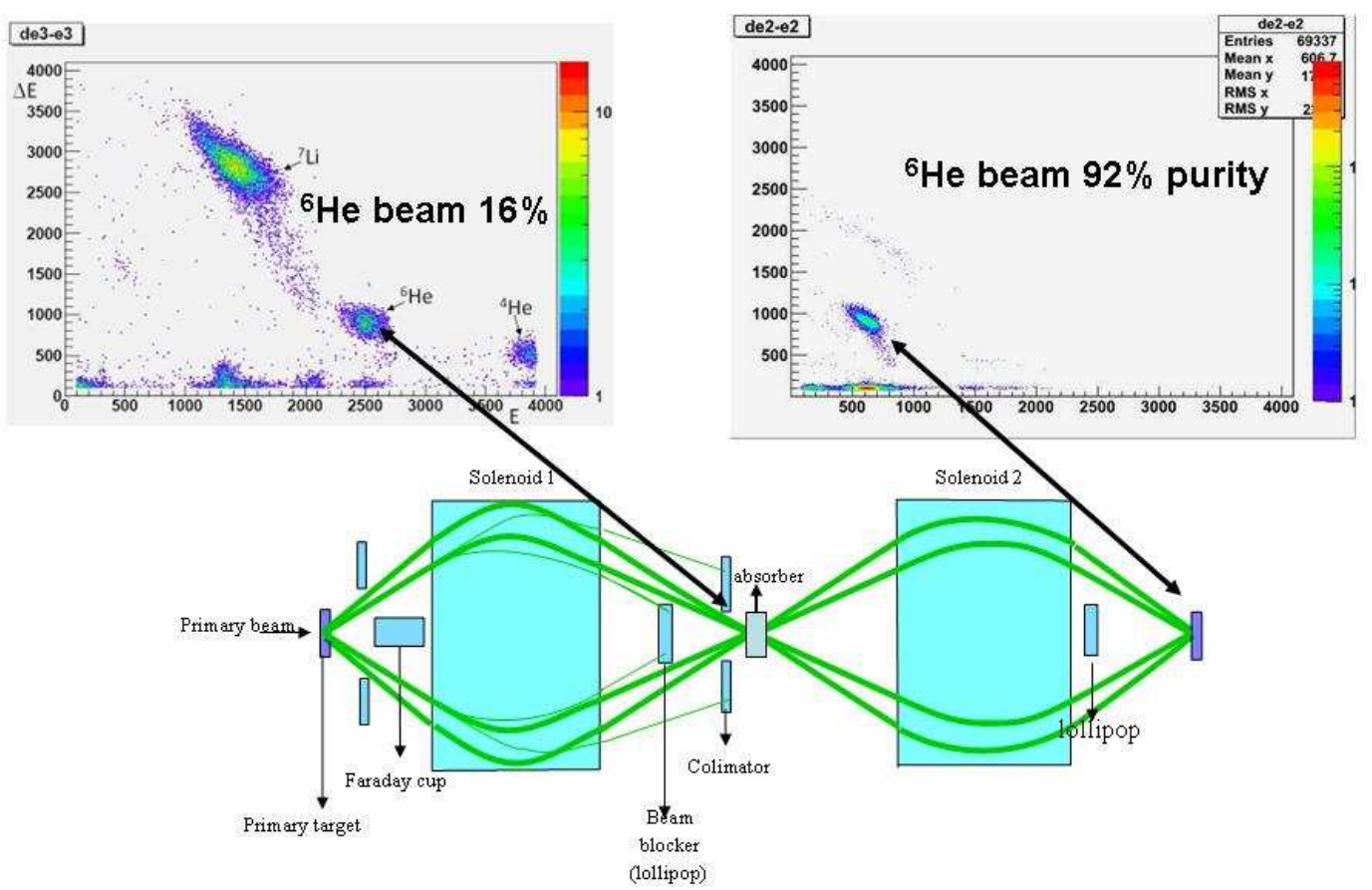

Figure 4: Spectrum of ${ }^{6} \mathrm{He}$ secondary beam in the intermediate and in the secondary scattering chamber after the second solenoid [21].

A preliminary analysis of this resonant peak has been performed by L. Canton [24] and is presented in the proceedings of the present conference.

\section{Recent and future developments}

Recent improvements have been implemented in the secondary scattering chamber. Two independent rotating plates have been installed (see figure 6) on which detectors can be fixed, permiting an independent adjustment of the detection angles. This system allows for kinematic coincidence measurements using large area strip detectors. All the mechanical system to control the movement of the plates has been developed in our laboratory by J. Diaz and M.A.G. Alvarez. The electronics and software control was developed by R. Diaz.

The movement of the plates is remotely controlled by two stepper motors and the measurement of the scattering angles is performed using the signals from reference sensors located under the plates, together with the angular scale marked on the plates, where the detector's supports are positioned. A target holder with 4 positions is placed in the center of the scattering chamber and the target position can be changed manually. The control of the position of the plates is made remotely without the need to break the vacuum in the chamber. 


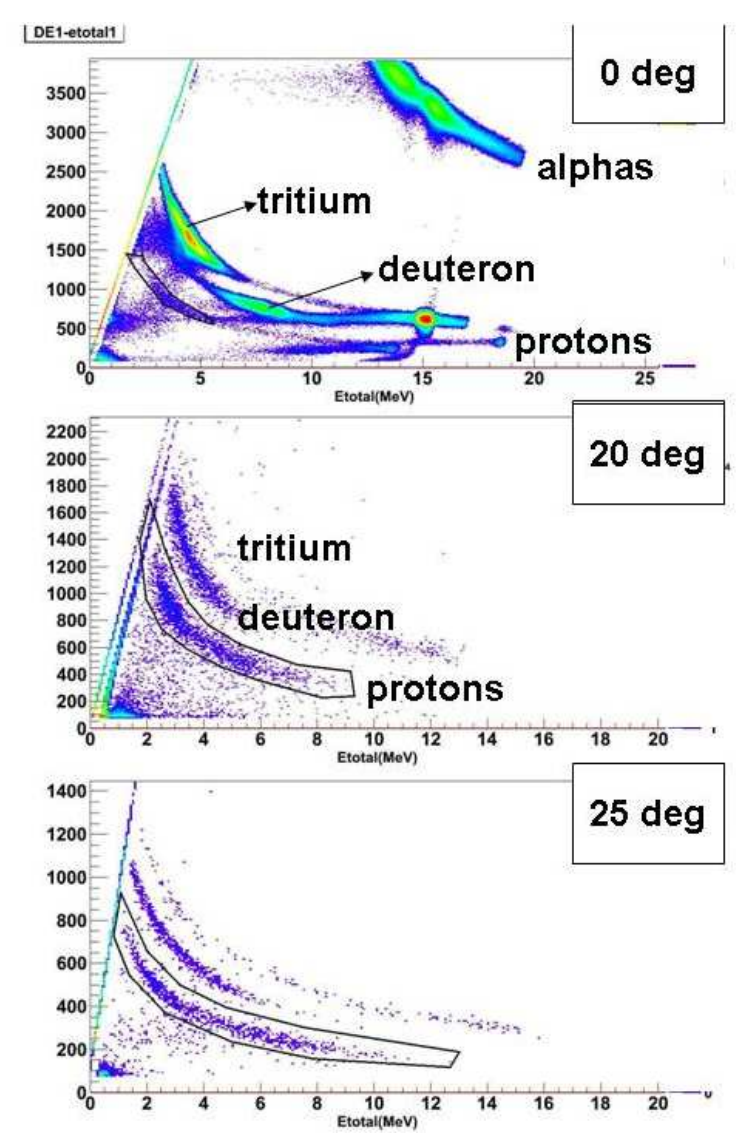

Figure 5: Spectra of light particles emitted from the collision of a ${ }^{6} \mathrm{He}$ beam on a $\mathrm{CH}_{2}$ target [23] at zero (top), 20 (mid) and 25 degrees (botton). The intense alpha, triton and deuterons peaks seen at zero degrees are contaminant beams. The poligonon indicate the region in the proton line where the $11.24 \mathrm{MeV}{ }^{7} \mathrm{Li}$ resonance should appear.

A new aquisition system based on VME and electronics has been installed to operate with the strip detectors. One doubled sided silicon strip detector (DSSD) of $40 \mu \mathrm{m}$, one single sided of $20 \mu \mathrm{m}$ which can be used as an energy loss $\Delta E$ detector, and one PAD of $1000 \mu \mathrm{m}$ for energy measurements are available.

We are planning to install a gamma cave in an extension of the beam line beyond the secondary scattering chamber, in a position where the residual magnetic field of the solenoid is sufficiently small to allow the use of gamma detectors. Gamma-particle coincidence measurements will then be possible.

\section{References}

[1] V. Efimov, Phys. Lett. B 33 (1970) 563.

[2] T. Kraemer, et al., Nature 440 (2006) 315.

[3] K. M. Burbidge, G. R. Burbidge, W. A. Fowler, F. Hoyle Rev. of Mod. Phys. vol 29, no. 4, 547, (1957).

[4] J. José and C. Iliadis, Rep. Progr. Phys. 74, 096901 (2011). 


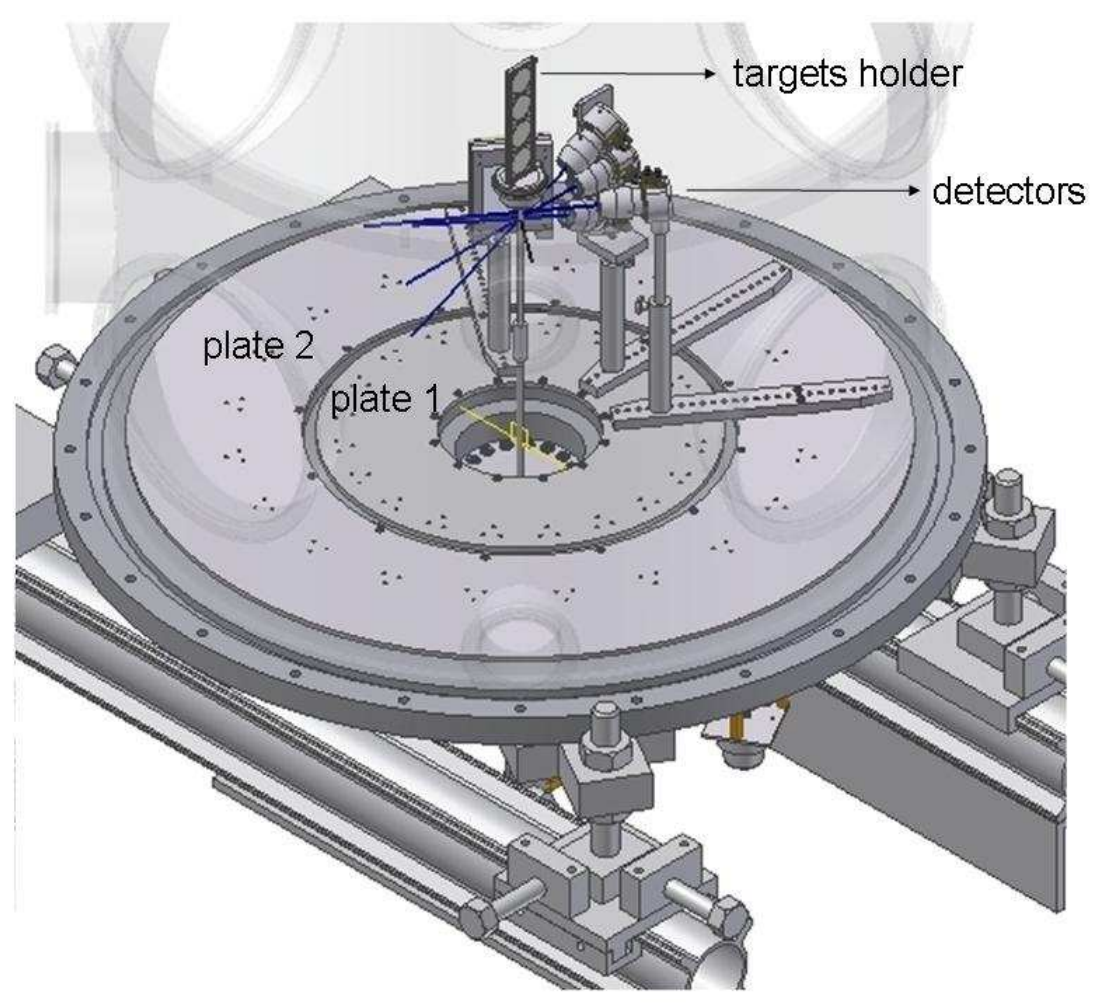

Figure 6: Rotating plates in the secondary scattering chamber after the second solenoid.

[5] J. Gorres, H. Herndl, I. J. Thompson, M. Wiescher, Phys. Rev. C52, 2231 (1995).

[6] R. Lichtenthäler, et al., Eur. Phys. J. A 25 (2005) 733.

[7] R. Lichtenthäler, et al., Nucl. Phys. News 15 (2005) 25.

[8] V. Morcelle, et al., Phys. Lett. B (2014) http://dx.doi.org/10.1016/j.physletb.2014.03.043

[9] E. A. Benjamim et al., Phys. Lett. B 647 (2007) 30.

[10] R. Lichtenthäler et al., Rev. Mex. Fis. 53, 59(2007).

[11] P. N. de Faria, et al., Phys. Rev. C 81 (2010) 044605.

[12] K. C. C. Pires, et al., Phys. Rev. C 83 (2011) 064603.

[13] S. Mukherjee et al., Eur. Phys. J. A 45, 23 (2010).

[14] A. Barioni et al., Phys. Rev. C 80, 034617 (2009). .

[15] J.C. Zamorra et al., Phys. Rev. C 84, 034611 (2011).

[16] P. N. de Faria, et al., Phys. Rev. C 82 (2010) 034602.

[17] P. Mohr et al., Phys. Rev. C 82 (2010) 044606. 
[18] Elastic Scattering Measurements for Be-7+Al-27 System at RIBRAS Facility Morcelle, V.; Lichtenthaeler, R.; Morais, M. C.; et al. Conference: 35th Brazilian Workshop on Nuclear Physics Location: Sao Sebastiao, BRAZIL Date: SEP 02-06, 2012 XXXV BRAZILIAN WORKSHOP ON NUCLEAR PHYSICS Book Series: AIP Conference Proceedings, vol.1529, 202(2013).

[19] D.R. Mendes Jr et al, Phys. Rev. C 86, 064321 (2012).

[20] V. Guimaraes, R. Lichtenthaeler, O. Camargo, A. Barioni, M. Assuncao, J. Kolata, H. Amro, F. D. Becchetti, H. Jiang, E. F. Aguilera, Phys. Rev. C75, 054602(2007).

[21] R. Lichtenthäler et al. XXXV BRAZILIAN WORKSHOP ON NUCLEAR PHYSICS Book Series: AIP Conference Proceedings, Volume: 1529, Pages: 197-201 (2013).

[22] E. Leistenschneider, A. Lépine-Szily et al., see procedings of the present conference.

[23] R. Pampa Condori et al., Proceedings of the INPC-2013 conference. EPJ Web of Conferences 66,03054 (2014)

[24] L. Canton, see proceedings of the present conference.

[25] D.R. Tilley et al., Nucl. Phys. A708, 3(2002). 